\title{
Widths of Isobaric Analog Resonances: a microscopic approach
}

\author{
G. Colò ${ }^{1}$, H. Sagawa ${ }^{2}$, N. Van Giai ${ }^{3}$, P.F. Bortignon ${ }^{1}$, and T. Suzuki ${ }^{4}$ \\ ${ }^{1}$ Dipartimento di Fisica, Università degli Studi and INFN, Sezione di Milano, Via Celoria 16, \\ 20133 Milano, Italy \\ ${ }^{2}$ Center for Mathematical Sciences, The University of Aizu, Aizu-Wakamatsu, Fukushima 965, \\ Japan \\ ${ }^{3}$ Division de Physique Théorique, Institut de Physique Nucléaire, 91406 Orsay Cedex, France \\ ${ }^{4}$ Department of Physics, College of Humanities and Sciences, Nihon University, Sakurajosui \\ 3-25-40, Setagaya-ku, Tokyo 156, Japan
}

(September 10, 2017)

\begin{abstract}
A self-consistent particle-phonon coupling model is used to investigate the properties of the isobaric analog resonance in ${ }^{208} \mathrm{Bi}$. It is shown that quantitative agreement with experimental data for the energy and the width can be obtained if the effects of isospin-breaking nuclear forces are included, in addition to the Coulomb force effects. A connection between microscopic model predictions and doorway state approaches which make use of the isovector monopole resonance, is established via a phenomenological ansatz for the optical potential.
\end{abstract}




\section{INTRODUCTION}

Since its discovery some 35 years ago, the isobaric analog resonance (IAR) has always attracted a considerable interest because it is one of the clearest manifestations of approximate isospin symmetry in atomic nuclei. The main causes of isospin symmetry breaking are essentially the long-range Coulomb force, and to a lesser extent the short-range nuclear forces of the charge-symmetry breaking (CSB) type and charge-independence breaking (CIB) type [1]. Quoting a sentence from a review paper on this subject [2], the Coulomb interaction "is strong enough so that its effects are quite easily detectable and weak enough not to destroy or alter considerably what the nuclear force has produced." Despite this favourable situation, we are still missing a microscopic model which is able to reproduce one of the physical observables related to the breaking of isospin symmetry in nuclei, namely the total width of the IAR, taking into account all the isospin-breaking effects.

The general features of the IAR are qualitatively well understood. In the absence of isospin-breaking effects, the analog state would be degenerate with the ground state of the $(N, Z)$ parent nucleus and it would have zero width. The effect of the Coulomb and other isospin-breaking forces is to shift up the analog state by several $\mathrm{MeV}$ (the so-called Coulomb displacement energy). However, these isospin-breaking forces do not induce strong isospin mixing of the states. The analog state, just as its parent state, has practically pure $T=T_{0}$ isospin $\left(T_{0} \equiv(N-Z) / 2\right)$ whereas the neighbouring states in the daughter nucleus have mostly $T=T_{0}-1$. Thus, a simple argument based on the Fermi golden rule shows that a small spreading width for the IAR must be expected. In addition, the escape width must also be small because the main escape channels are those of low energy protons.

When one attempts to build a microscopic model of IAR, the requirement that isospin symmetry must be restored if isospin-breaking forces are switched off should be taken into account. It is known that in $N \neq Z$ systems the Hartree-Fock (HF) approximation introduces a spurious isospin symmetry breaking and that a self-consistent charge-exchange random phase approximation (RPA) restores this broken symmetry [3]. By self-consistent 
RPA we mean that the HF single-particle spectrum and the residual particle-hole interaction are derived from the same effective two-body force. Therefore, we shall consider here only this self-consistent framework. Charge-exchange RPA was applied for the first time to IAR studies in Ref. [4] (see also [2] and references therein). Calculations of other types of excitations using charge-exchange RPA can be found in Ref. [5]. However, the RPA description can at best give information on escape widths if continuum effects are included but it cannot shed any light on spreading widths because this spreading is due to states beyond the one particle-one hole $(1 \mathrm{p}-1 \mathrm{~h})$ space. An extension of the model space to include $2 \mathrm{p}-2 \mathrm{~h}$ configurations and leading to second RPA would be a more appropriate scheme. In heavy nuclei where the neutron excess is large one can safely replace the RPA by the Tamm-Dancoff approximation (TDA). In Ref. [6] such a second TDA calculation was performed for the IAR in ${ }^{208} \mathrm{Bi}$ and it gave a reasonable estimate of the escape width $\Gamma^{\uparrow}$ and spreading width $\Gamma^{\downarrow}$. More recently, calculations based on a particle-phonon coupling model [7] also led to satisfactory values of $\Gamma^{\uparrow}$ and $\Gamma^{\downarrow}$ but some discrepancies with experimental data still remain. In the above mentioned works, only isospin breaking due to the inclusion of the Coulomb force in the HF mean field was considered.

The purpose of the present paper is twofold. Firstly, we wish to examine the effects of the isospin-breaking nuclear forces on the properties of the IAR. It is known that the CSB and CIB interactions affect the mass number dependence of the Coulomb displacement energy anomalies and bring them in better agreement with experiment [8]. Moreover, these isospin-breaking nuclear forces lead to an increase of about $50 \%$ of the isospin mixing in nuclear ground states [9]. Thus, one should expect also some sizable effects on the values of $\Gamma_{I A R}^{\downarrow}$. We study these effects within the particle-phonon coupling model [7] together with the short range parametrizations of the CSB and CIB forces of Ref. [9]. The calculations are performed for the typical case of ${ }^{208} \mathrm{Bi}$. Secondly, it is instructive to study the connection between a microscopic model such as the present one or the second TDA model of Ref. [6], and IVMR doorway state approaches [10,11] where the shift and width of the IAR result from the coupling via the Coulomb interaction of an ideal analog state with the isovector 
monopole resonance (IVMR) playing the role of a doorway state. Here, we show that this connection can be established if one makes a phenomenological ansatz for the isospin dependence of the nucleon optical potential.

In Sect. II the microscopic model is presented and its results are discussed in Sect. III. The connection between the microscopic model and the approach of Ref. [1] is shown in Sect. IV. Conclusions are drawn in Sect. V.

\section{THE MICROSCOPIC MODEL}

The RPA extended so as to include the continuum coupling and the particle-phonon coupling has been described in detail in Ref. [7] and therefore, we shall first recall only the main features of the model and then proceed to the specific points of the present calculation.

Starting from an effective Hamiltonian $H$ with two-body Skyrme interaction [12, 13, Coulomb interaction and CSB-CIB interactions which will be described below, the HF equations determine the self-consistent mean field of the parent nucleus. This mean field is diagonalized on a basis of 15 shells of harmonic oscillator wave functions $\left(\hbar \omega_{\text {osc }}=6.2\right.$ $\mathrm{MeV}$ for ${ }^{208} \mathrm{~Pb}$ ). This procedure provides a discrete set of levels (occupied and unoccupied). We select all occupied levels, and 6 unoccupied levels for each value of $(l, j)$ with increasing values of the radial quantum number $n$. This determines the subspace $Q_{1}$ of discrete $1 \mathrm{p}-1 \mathrm{~h}$ (proton particle-neutron hole) configurations.

To account for the escape width $\Gamma^{\uparrow}$ and spreading width $\Gamma^{\downarrow}$ of the IAR, we build two other subspaces $P$ and $Q_{2}$. The space $P$ is made of particle-hole configurations where the particle is in an unbound state orthogonal to all the above discrete single-particle levels. The method to calculate these unbound states is described in Ref. [7]. On the other hand, the space $Q_{2}$ is built with the main configurations which are known to play a major role in the damping process of nuclear giant resonances: these configurations are $1 \mathrm{p}-1 \mathrm{~h}$ states coupled to a collective vibration. We have included in our model space all the isoscalar vibrations of multipolarity $2^{+}, 3^{-}$and $4^{+}$up to the energy of $20 \mathrm{MeV}$ and which exhaust more than $1 \%$ 
of the energy-weighted sum rule (EWSR) of the corresponding multipole operator. These collective vibrations are calculated consistently in HF-RPA within the $Q_{1}$ space and they constitute the phonons of our particle-phonon coupling model.

Using the projection operator formalism one can easily find that the effects of coupling the subspaces $P$ and $Q_{2}$ to $Q_{1}$ are described by the following effective Hamiltonian acting in the $Q_{1}$ space:

$$
\begin{aligned}
& \mathcal{H}(E) \equiv Q_{1} H Q_{1}+W^{\uparrow}(E)+W^{\downarrow}(E) \\
= & Q_{1} H Q_{1}+Q_{1} H P \frac{1}{E-P H P+i \epsilon} P H Q_{1}+Q_{1} H Q_{2} \frac{1}{E-Q_{2} H Q_{2}+i \epsilon} Q_{2} H Q_{1},
\end{aligned}
$$

where $E$ is the excitation energy. For each value of $E$ the RPA equations corresponding to this effective, complex Hamiltonian $\mathcal{H}(E)$ are solved. The resulting sets of eigenstates enable one to calculate all relevant quantities such as giant resonances energies and widths (see Ref. [0]). In practice, we use the HF-RPA states (corresponding to positive and negative eigenvalues) as a basis for the $Q_{1}$ space because we can truncate this basis and neglect states which have negligible $T_{-}$strength.

A simplifying approximation is made when calculating the matrix elements of $W^{\downarrow}$ by neglecting the interactions among the states within $Q_{2}$. Each matrix element $W_{p h, p^{\prime} h^{\prime}}^{\downarrow}$ is a sum of four terms whose diagrammatic representation is shown in Fig. 1. To evaluate these diagrams we use the following expression for the particle-vibration vertices:

$$
V=\sum_{\alpha \beta} \sum_{L n M}\left\langle\alpha\left|\varrho_{n}^{(L)}(r) v(r) Y_{L M}(\hat{r})\right| \beta\right\rangle a_{\alpha}^{\dagger} a_{\beta}
$$

This form comes from the particle-vibration coupling model where the vibration (phonon) $|n\rangle$ is characterized by its angular momentum $L$ and its radial transition density $\varrho_{n}^{(L)}(r)$. The form factor $v(r)$ appearing in (2) is related to the particle-hole interaction derived from the Skyrme force by $V_{p h}\left(\vec{r}_{1}, \vec{r}_{2}\right)=v\left(r_{1}\right) \delta\left(\vec{r}_{1}-\vec{r}_{2}\right)$. When deriving this form factor, the velocity-dependent terms of the residual Skyrme interaction are dropped. 
It was pointed out in Ref. [7] that the particle-phonon coupling model does not automatically insure the isospin symmetry properties of the nuclear forces (contrarily to a fully microscopic $2 \mathrm{p}-2 \mathrm{~h}$ model like in Ref. [6]). One must therefore enforce isospin symmetry in the evaluation of $W_{p h, p^{\prime} h^{\prime}}^{\downarrow}$ by an appropriate isospin projection procedure [7].

In the present calculations the Skyrme interactions SIII [12] and SGII [13] have been employed for the isospin symmetric part of the Hamiltonian. In addition, CSB and CIB effective nucleon-nucleon forces determined in Ref. [9] are also included. These forces were obtained from a short-range expansion of Yukawa-type interactions and they have a form similar to that of Skyrme forces:

$$
\begin{aligned}
V_{C S B}= & \frac{1}{4}\left\{\tau_{z}(1)+\tau_{z}(2)\right\}\left\{s_{0}\left(1+y_{0} P_{\sigma}\right)+\frac{1}{2} s_{1}\left(1+y_{1} P_{\sigma}\right)\right. \\
& \left.\times\left(\vec{k}^{2}+\vec{k}^{\prime 2}\right)+s_{2}\left(1+y_{2} P_{\sigma}\right) \vec{k}^{\prime} \cdot \vec{k}\right\}
\end{aligned}
$$

and

$$
\begin{aligned}
V_{C I B}= & \frac{1}{2} \tau_{z}(1) \tau_{z}(2)\left\{u_{0}\left(1+z_{0} P_{\sigma}\right)+\frac{1}{2} u_{1}\left(1+z_{1} P_{\sigma}\right)\right. \\
& \left.\times\left(\vec{k}^{2}+\vec{k}^{\prime 2}\right)+u_{2}\left(1+z_{2} P_{\sigma}\right) \vec{k}^{\prime} \cdot \vec{k}\right\}
\end{aligned}
$$

The parameters $s_{i}$ and $u_{i}$ are given in Ref. [9], and all exchange parameters $y_{i}$ and $z_{i}$ are -1 because of the singlet-even character of $V_{C S B}$ and $V_{C I B}$. Therefore, they do not contribute as residual particle-hole interactions in the isovector channel and their only influence is through their contributions to the HF mean field.

\section{DISCUSSION OF RESULTS}

Three types of calculations have been done which are labeled by I, II, III respectively. In calculation I the starting Hamiltonian contains only the Skyrme interaction without the Coulomb force between protons and without CSB-CIB nuclear forces. In calculation II the Coulomb interaction is also included. In calculation III the CSB-CIB forces are added. Thus,

the three calculations have an increasing degree of isospin breaking and they are expected 
to lead to increasing values of the IAR width according to the arguments recalled before. In calculations II and III the Coulomb exchange contributions to the mean field are treated in the Slater approximation whereas the Coulomb p-h residual interaction is dropped. The results obtained by using respectively the interactions SIII and SGII are shown in Tables 1 and 2. Calculation II is in principle equivalent to what was done in Ref. [7] except for two changes in the model spaces $P$ and $Q_{2}$, and in the averaging parameter $\Delta$. Because of these changes, the values of the mean energies and widths of the IAR we quote as calculation II are slightly different from the correspnding values reported in [7]. The spaces $P$ and $Q_{2}$ have been enlarged with respect to the calculation of Ref. [7] by including a larger (96 instead of 64) number of unoccupied proton single-particle states. This affects mainly the escape and spreading widths calculated with the interaction SIII which both increase by about 10 $\mathrm{keV}$ whereas the widths calculated with the interaction SGII are less affected. Also, the averaging parameter $\Delta$ (see Ref. [7) employed in the calculation has been changed in the present work from $100 \mathrm{keV}$, as it was in [7], to $200 \mathrm{keV}$. In fact, we have carefully studied the dependence of the spreading width on the parameter $\Delta$, and we have found that $\Gamma^{\downarrow}$ increases by about $20 \mathrm{keV}$ when $\Delta$ is changed from $100 \mathrm{keV}$ to $200 \mathrm{keV}$ (as it was already discussed in Ref. [7]) and also increases by another $20 \mathrm{keV}$ when $\Delta$ is changed from $200 \mathrm{keV}$ to 400 $\mathrm{keV}$. On the other hand, $\Gamma^{\downarrow}$ remains constant if $\Delta$ is set above $400 \mathrm{keV}$. Therefore, we have adopted the value of $200 \mathrm{keV}$ for $\Delta$ since this value is intermediate between $0 \mathrm{keV}$ and 400 $\mathrm{keV}$ (the value at which $\Gamma^{\downarrow}$ saturates). This study allows us to say that the uncertainity on the values of $\Gamma^{\downarrow}$ due to the freedom of choice of the parameter $\Delta$ is about $\pm 20 \mathrm{keV}$.

The calculation without any isospin-breaking force (calculation I) has been performed to show that our procedure can recover the isospin symmetry reasonably well at each step. The first step (a) is discrete TDA since discrete RPA is not possible in this case because of the negative energy configurations. Indeed, without the Coulomb interaction the neutron states lie higher in energy than their proton partners and the energies $\varepsilon_{p}-\varepsilon_{h}$ of the unperturbed proton particle-neutron hole $0^{+}$configurations are negative. For the six excess neutron hole levels and the corresponding proton particle levels, these values are between $-7.3 \mathrm{MeV}$ 
and -8.3 MeV in the case of the force SGII and between -8.6 MeV and $-10 \mathrm{MeV}$ in the case of the force SIII. In an ideally accurate numerical calculation these negative energy configurations would be coherently pushed up to form a collective state at zero energy. Small numerical inaccuracies can weaken the residual interaction, and as a result complex RPA eigenvalues may appear. This can be easily understood in the simple case of a schematic model, or in a case containing a single state, where the RPA and TDA energies are related by $E_{R P A}^{2}=E_{T D A}^{2}-V^{2}$ with $V$ representing the interaction term. In the limit $V_{C}=0$ the r.h.s. may become slightly negative and consequently $E_{R P A}$ is imaginary, if $E_{T D A}^{2}$ and $V^{2}$ do not exactly cancel numerically. This difficulty of complex RPA solutions in the case $V_{C}=0$ is well known and, for example, in Ref. [2] the way out was to slightly renormalize the nuclear residual interaction. Therefore, only TDA is possible for the study of the IAR without the Coulomb interaction. One should also note that, contrary to a naive view, adding the Coulomb interaction does not result in an overall shift of the RPA eigenvalues. This can again be seen, e.g., in the framework of a schematic model. The second step (b) is RPA with the coupling to the continuum, which gives no width since the IAR is below the proton emission threshold. The aim of the third step (c) (inclusion of the spreading width) is to know whether the particle-phonon coupling model we have adopted can introduce some spurious width because of the approximations made: finite size of the set of $1 \mathrm{p}-1 \mathrm{~h}$ plus phonon states, overcompleteness of the $1 \mathrm{p}-1 \mathrm{~h}$ plus phonon basis, violation of the Pauli principle, simple form of the particle-phonon vertex function. The fact that we obtain only $4 \mathrm{keV}$ for the spurious $\Gamma^{\downarrow}$ with the force SGII is very satisfactory since it means that the approximations we have mentioned are safe in this case.

In the case of the force SIII the spurious width $(24 \mathrm{keV})$ is larger than that obtained with the force SGII. We have reached the conclusion that this spuriosity is due by half to the particle-phonon coupling and by half to the fact that HF-TDA is not completely able to recover the isospin symmetry. To estimate the spuriosity due to the particle-phonon coupling, we have considered an ideal analog state at zero energy with the following schematic wave function: 


$$
|A\rangle=\frac{1}{\sqrt{2 T_{0}}} \hat{T}_{-}|H F\rangle=\sum_{\pi, \nu^{-1}} X_{\pi, \nu^{-1}}\left|\pi, \nu^{-1}\right\rangle
$$

with $\nu^{-1}$ restricted to the excess neutrons and

$$
X_{\pi, \nu^{-1}}=\frac{1}{\sqrt{2 T_{0}}} \delta\left(l_{\pi}, l_{\nu}\right) \delta\left(j_{\pi}, j_{\nu}\right) \sqrt{2 j_{\pi}+1} \int d r u_{\pi}(r) u_{\nu}(r) .
$$

We have calculated the coupling of this schematic IAR with the $1 \mathrm{p}-1 \mathrm{~h}$ plus phonon states adopted for all the calculations of Table I, and we have obtained a state at $27.6 \mathrm{keV}$ whose width is $13 \mathrm{keV}$. Therefore, $13 \mathrm{keV}$ is the broadening of the IAR introduced spuriously by the coupling with particle-hole-phonon configurations. The remaining $11 \mathrm{keV}$ is still larger than the value obtained with the interaction SGII. This is on one hand related to the different single-particle levels obtained for the two interactions. As mentioned above, proton particle and neutron hole levels are more separated in the case of the force SIII. Consequently, the isospin breaking in the HF field is restored less efficiently by TDA and the energy of the IAR is less close to zero than in the case of the force SGII. Therefore, in the case of SIII we diagonalize the effective Hamiltonian (1) at a higher value of the energy $E$ and the spreading width of the IAR at higher excitation energy is larger. Moreover, the imaginary parts of the self-energy terms in our model would cancel exactly to give a zero spreading width if the single-particle radial wave functions of neutrons and protons with the same quantum numbers $(n, l, j)$ were identical. This cancellation can be seen by looking at the expressions of the four diagrams of Fig. 1 [7]. However, the cancellation is not complete if the singleparticle radial wave functions are different. The difference between radial wave functions is larger in the case of the force SIII, so again the spurious width is expected to be larger than that of SGII.

If we now include the Coulomb interaction between protons in the HF mean field, the proton levels are pushed up and become higher than the corresponding neutron levels. The energies of the unperturbed proton particle-neutron hole $0^{+}$configurations have positive values between 11 and $11.8 \mathrm{MeV}$ for SGII and between 8.8 and $10.3 \mathrm{MeV}$ for SIII (for the six main configurations already considered above). This difference between the two forces is 
essentially related to the fact that in the case of the force SGII the neutron holes are more bound. Therefore, the proton particles which enter the IAR wave function have less energy available $\left(\varepsilon_{p}=E_{I A R}+\varepsilon_{h}\right)$ and a smaller probability to escape. This explains why the $\Gamma^{\uparrow}$ is considerably smaller than in the case of the force SIII.

Finally, the most important result of our calculation is that the total width obtained by employing the force SIII (in the case of the complete calculation III, last column of Table 1) nicely agrees with the experimental finding $\Gamma_{T O T}^{(\exp )}=230 \mathrm{keV}$. The improvement with respect to calculation II (without CSB-CIB forces) is about 15\%. This shows that CSB-CIB forces can contribute significantly to the total width of the IAR.

\section{COMPARISON WITH THE IVMR DOORWAY STATE APPROACH}

We have seen that microscopic approaches, like the particle-phonon coupling model described above or the $2 \mathrm{p}-2 \mathrm{~h}$ TDA model of Ref. [6] can give a reasonable description of IAR widths in spite of some sensitivity to the effective interactions. The spreading widths come from the coupling terms $Q_{1} H Q_{2}$ appearing in $W^{\downarrow}$ of Eq. (1), and this coupling between the simple $Q_{1}$ configurations and more complex $Q_{2}$ configurations is produced mostly by the isospin-conserving Skyrme interaction (in fact, this is the only residual interaction we keep in the calculation of $W^{\downarrow}$ in the previous sections). Thus, the fact that the resulting $\Gamma^{\downarrow}$ is non-zero is due entirely to the effects of the Coulomb and other isospin-breaking forces in the mean field as they produce a finite density of states with the isospin of the parent nucleus at the IAR energy. On the other hand, in the approaches of Refs. [10,2, 11] $\Gamma^{\downarrow}$ originates from the coupling of an ideal analog state $|A\rangle$ with a specific doorway state, namely the IVMR in the daughter nucleus, via the isospin-breaking part of the Hamiltonian (usually the isovector component of the Coulomb force). Here, we show that these apparently different points of view can be connected.

Let us first recall the expression for $\Gamma^{\downarrow}$ obtained in Ref. [11]. The Hamiltonian is assumed 
to be a sum of an isospin-conserving part plus the Coulomb interaction, $H=H_{0}+V_{C}$, and the parent ground state which is eigenstate of $H_{0}$ is denoted by $|0\rangle$. The three isospin components of the IVMR in the daughter nucleus are schematically written as

$$
\left|M ; T_{0}+i, T_{0}-1\right\rangle=\left|\left\{|0\rangle^{T=T_{0}} \otimes\left|p h^{-1}\right\rangle^{T=1}\right\}_{T_{0}-1}^{T+i}\right\rangle
$$

where $i=-1,0,1$ and $\left|p h^{-1}\right\rangle$ stands for a combination of monopole p-h excitations. In Ref. 11] the IAR spreading width $\tilde{\Gamma}^{\downarrow}$ (we use this notation for the spreading width calculated by following the doorway state approach) was expressed in terms of the analog state energy $E_{A}$, IVMR energies $E_{M}^{T_{0}+i}$, the width $\Gamma_{M}\left(E_{A}\right)$ of the IVMR evaluated at energy $E=E_{A}$, and the reduced Coulomb matrix element:

$$
\tilde{v}_{C}=\frac{1}{\sqrt{3}}\left\langle\left(p h^{-1}\right)^{T=1}\left\|V_{C}^{(1)}\right\| 0\right\rangle
$$

where $V_{C}^{(1)}$ is the isovector part of the Coulomb potential. If one neglects the isospin splittings of the IVMR and adopts a common value $E_{M}^{T_{0}+i} \simeq E_{M}$ the expression of $\tilde{\Gamma}^{\downarrow}$ takes the simple form

$$
\tilde{\Gamma}_{A}^{\downarrow}=\Gamma_{M}\left(E_{A}\right) \frac{\left|\tilde{v}_{C}\right|^{2}}{\left(E_{A}-E_{M}\right)^{2}+\left(\frac{\Gamma_{M}}{2}\right)^{2}} .
$$

Furthermore, it was shown that the isospin mixing probability of the $T_{0}+1$ component of IVMR in the parent ground state $|\pi\rangle$ is given in second order perturbation theory by

$$
\begin{aligned}
\left|c_{T_{0}+1}\right|^{2} & \equiv \frac{1}{2\left(T_{0}+1\right)}\left\langle\pi\left|T_{-} T_{+}\right| \pi\right\rangle \\
& =\frac{1}{T_{0}+1} \frac{\left|\tilde{v}_{C}\right|^{2}}{\left|\Delta E_{M}\right|^{2}}
\end{aligned}
$$

where $\Delta E_{M}$ is the excitation energy of the IVMR in the parent nucleus which is approximately equal to $E_{A}-E_{M}$ of Eq. (9). We can safely neglect $\Gamma_{M}$ in Eq. (9) and thus obtain

$$
\tilde{\Gamma}_{A}^{\downarrow} \sim \frac{1}{2} \Gamma_{M}\left(E_{A}\right)\left\langle\pi\left|T_{-} T_{+}\right| \pi\right\rangle
$$

In the microscopic models [6,7] the spreading width of the IAR results from the couplings mediated by the isospin-conserving operator $W^{\downarrow}$ defined in section II. Denoting the RPA eigenstate corresponding to the IAR by $|A\rangle$, we can write its width as 


$$
\Gamma_{A}^{\downarrow}=-2 \operatorname{Im}\left\langle A\left|W^{\downarrow}\right| A\right\rangle .
$$

The IAR wave function can be well approximated by

$$
|A\rangle=\frac{1}{\sqrt{2 T_{0}}} T_{-}|\pi\rangle
$$

For the isospin-conserving $W^{\downarrow}$ interaction we make the ansatz:

$$
\begin{aligned}
W^{\downarrow} & =a(\vec{T} \cdot \vec{T}-b) \\
& =\left(a_{R}+i a_{I}\right)(\vec{T} \cdot \vec{T}-b),
\end{aligned}
$$

and obtain

$$
\begin{aligned}
\left\langle A\left|W^{\downarrow}\right| A\right\rangle & =\frac{a}{2 T_{0}}\left\langle\pi\left|T_{+}(\vec{T} \cdot \vec{T}-b) T_{-}\right| \pi\right\rangle \\
& =\frac{a}{2 T_{0}}\left\langle\pi\left|T_{+} T_{-}(\vec{T} \cdot \vec{T}-b)\right| \pi\right\rangle \\
& \approx \frac{a}{2 T_{0}}\left\langle\pi\left|T_{+} T_{-}\right| \pi\right\rangle\langle\pi|(\vec{T} \cdot \vec{T}-b)| \pi\rangle .
\end{aligned}
$$

In the last step of the above equation the contributions of the excited states to the closure relation have been dropped because they are of order $\left|c_{T_{0}+1}\right|^{2}$. Let us introduce $\tilde{T}$ by:

$$
\langle\pi|\vec{T} \cdot \vec{T}| \pi\rangle \equiv \tilde{T}(\tilde{T}+1)
$$

( $\tilde{T}$ differs slightly from $T_{0}$ because $|\pi\rangle$ has isospin mixing) and choose $b=\tilde{T}^{2}$. Then:

$$
\left\langle A\left|W^{\downarrow}\right| A\right\rangle=a[\tilde{T}(\tilde{T}+1)-b]=a \tilde{T}
$$

Thus, the two expressions (11) and (12) are equal if the following condition is satisfied:

$$
a_{I}=-\frac{\Gamma_{M}\left(E_{A}\right)}{4 \tilde{T}}\left\langle\pi\left|T_{-} T_{+}\right| \pi\right\rangle
$$

Next, we diagonalize the TDA schematic model with the interaction

$$
v_{e f f}=\left(a_{R}+i a_{I}\right)(\vec{T} \cdot \vec{T}-b)
$$

The complex eigenvalue is 


$$
\begin{aligned}
E_{A}-i \frac{\Gamma_{A}}{2} & =\varepsilon_{p h}+\frac{v_{e f f}}{2} \sum_{p h}\left|\left\langle\left(p h^{-1}\right)^{T=1, T_{z}=-1}\left|T_{-}\right| 0\right\rangle\right|^{2} \\
& =\varepsilon_{p h}+\frac{a_{R}}{2} \cdot 2 T_{0}+i \frac{a_{I}}{2} \cdot 2 T_{0},
\end{aligned}
$$

where $\varepsilon_{p h}$ is the degenerate unperturbed energy of the $0^{+}$proton particle-neutron hole configurations. Replacing $a_{I}$ by its value (18) we see that $\Gamma_{A}$ becomes

$$
\Gamma_{A}=\frac{1}{2} \Gamma_{M}\left(E_{A}\right) \frac{T_{0}}{\tilde{T}}\left\langle\pi\left|\hat{T}_{-} \hat{T}_{+}\right| \pi\right\rangle
$$

which is consistent with Eq. (11). Thus, the adopted interaction $W^{\downarrow}$ results in a value of

the spreading width coming from the coupling of the IAR with states of $2 \mathrm{p}-2 \mathrm{~h}$ type through the nuclear interaction, which is comparable with the result of the IVMR doorway state approach in which the spreading width is obtained through the coupling of the IAR to the IVMR due to the Coulomb interaction. It is left as a future problem the justification of the choice of (14) from a microscopic study of optical potentials.

\section{CONCLUSION}

Within the framework of a microscopic model based on self-consistent HF-RPA plus coupling with continuum configurations as well as with $1 \mathrm{p}-1 \mathrm{~h}$ plus phonon configurations, we have calculated the total width of the IAR in ${ }^{208} \mathrm{Bi}$. We have shown that if the nuclear isospin-breaking forces of CSB and CIB type are included in the Hamiltonian in addition to the Coulomb interaction, the width of the IAR is increased by 15-20\%. Thus, the nuclear isospin-breaking interactions which were already known to increase the isospin mixing of ground states have also significant contributions to the total width of the IAR. As far as comparison with experiment is concerned, the values of $\Gamma^{\downarrow}$ and $\Gamma_{T O T}$ calculated with SIII are in satisfactory agreement with the data whereas those obtained with SGII are not so good due to the peculiarities of the single-particle spectra of SGII.

Our microscopic model introduces some spuriosity in the evaluation of the total width of the IAR. This spuriosity turns out to be quite small and we have shown that it is due partly to 
the incomplete restoration of symmetry by TDA and partly to the $1 \mathrm{p}-1 \mathrm{~h}$ plus phonon model. Indeed, this model does not have the full self-consistency of a second RPA calculation which would be free in principle of spurious isospin violations. However, a second RPA calculation including also continuum effects would be extremely difficult and it has never been done so far.

Finally, we have been able to propose for the first time a connection between the microscopic model and IVMR doorway state approaches for the spreading width of the IAR. This

connection is possible by making a phenomenological ansatz for the isospin dependence of the nucleon optical potential.

\section{ACKNOWLEDGMENTS}

G.C. likes to acknowledge the nice hospitality of the Division de Physique Théorique (IPN, Orsay) where part of the work has been done. H.S. and P.F.B. acknowledge the warm hospitality of the Institute for Nuclear Theory of Seattle, where this project started during the 1995 workshop on nuclear structure. 


\section{REFERENCES}

[1] E.M. Henley and G.A. Miller, in Mesons in Nuclei, Vol. 1, eds. M. Rho and D.H. Wilkinson (North Holland, Amsterdam, 1979).

[2] N. Auerbach, Phys. Rep. 98, 273 (1983).

[3] C.A. Engelbrecht and R.H. Lemmer, Phys. Rev. Lett. 24, 607 (1970); A.M. Lane and J. Martorell, Ann. Phys. (N.Y.) 129, 273 (1980).

[4] N. Auerbach, N. Van Giai and A. Yeverechyahu, in Highly Excited States in Nuclear Reactions, eds. H. Ikegami and M. Muraoka (RCNP, Osaka, 1980), p. 623.

[5] N. Auerbach and A. Klein, Nucl. Phys. A395, 77 (1983).

[6] S. Adachi and S. Yoshida, Nucl. Phys. A462, 61 (1987).

[7] G. Colò, N. Van Giai, P.F. Bortignon and R.A. Broglia, Phys. Rev. C 50, 1496 (1994).

[8] T. Suzuki, H. Sagawa and N. Van Giai, Phys. Rev. C 47, 1360 (1993).

[9] H. Sagawa, N. Van Giai and T. Suzuki, Phys. Lett. B 353, 7 (1995).

[10] A.Z. Mekjian, Phys. Rev. Lett. 25, 888 (1970).

[11] T. Suzuki, H. Sagawa and G. Colò, Phys. Rev. C 54, 2954 (1996).

[12] M. Beiner, H. Flocard, N. Van Giai and P. Quentin, Nucl. Phys. A238, 29 (1975).

[13] N. Van Giai and H. Sagawa, Phys. Lett. B 106, 379 (1981). 


\section{FIGURES}

FIG. 1. Diagrams corresponding to the coupling of the $1 \mathrm{p}-1 \mathrm{~h}$ configurations to the more complicated states including a phonon (wavy line). The sum of the four diagrams gives the matrix element $\mathrm{W}_{p h, p^{\prime} h^{\prime}}^{\downarrow}$ quoted in the text. 


\section{TABLES}

TABLE I. IAR results with the interaction SIII. Three different types of interactions based on SIII are used in the calculations: I) Skyrme interaction without Coulomb force; II) Skyrme interaction with Coulomb force; III) Skyrme interaction with Coulomb force and CSB-CIB forces. Three different microscopic models are also adopted: a) TDA without the coupling to the continuum; b) RPA with the coupling to the continuum; c) RPA with the couplings to both the continuum and the phonons. Energies are given in $\mathrm{MeV}$ and widths are in $\mathrm{keV}$. The percentage of total strength $\mathrm{m}_{0}=(\mathrm{N}-\mathrm{Z}) / 2$ exhausted by the IAR is also shown.

\begin{tabular}{|c|c|c|c|c|c|c|c|c|c|}
\hline & \multicolumn{2}{|c|}{ (a) Discrete TDA } & \multicolumn{3}{|c|}{ (b) $\mathrm{RPA}+\mathrm{W}^{\uparrow}$} & \multicolumn{4}{|c|}{ (c) $\mathrm{RPA}+\mathrm{W}^{\uparrow}+\mathrm{W}^{\downarrow}$} \\
\hline & $\mathrm{E}_{I A R}$ & $\%$ of $\mathrm{m}_{0}$ & $\mathrm{E}_{I A R}$ & $\Gamma^{\uparrow}$ & $\%$ of $\mathrm{m}_{0}$ & $\mathrm{E}_{I A R}$ & $\Gamma_{T O T}$ & $\Gamma^{\downarrow}$ & $\%$ of $\mathrm{m}_{0}$ \\
\hline I & 0.268 & 99.9 & - & - & - & 0.267 & 24 & 24 & 99.7 \\
\hline \multirow[t]{2}{*}{ II } & 18.50 & 85 & 18.50 & 124 & 97 & 18.36 & 194 & 70 & 97 \\
\hline & 18.28 & 16 & & & & & & & \\
\hline \multirow[t]{2}{*}{ III } & 18.64 & 80 & 18.65 & 128 & 96 & 18.54 & 228 & 100 & 96 \\
\hline & 18.39 & 11 & & & & & & & \\
\hline
\end{tabular}


TABLE II. IAR results obtained with the interaction SGII. For details, see the caption to the previous table.

\begin{tabular}{cccccccccc}
\hline \hline & \multicolumn{2}{c}{ (a) Discrete TDA } & \multicolumn{2}{c}{ (b) RPA $+\mathrm{W}^{\uparrow}$} & \multicolumn{3}{c}{ (c) RPA $+\mathrm{W}^{\uparrow}+\mathrm{W}^{\downarrow}$} \\
\hline & $\mathrm{E}_{I A R}$ & \% of $\mathrm{m}_{0}$ & $\mathrm{E}_{I A R}$ & $\Gamma^{\uparrow}$ & \% of $\mathrm{m}_{0}$ & $\mathrm{E}_{I A R}$ & $\Gamma_{T O T}$ & $\Gamma^{\downarrow}$ & $\%$ of $\mathrm{m}_{0}$ \\
\hline I & 0.185 & 99.8 & - & - & - & 0.185 & 4 & 4 & 99.8 \\
II & 18.50 & 87 & 18.61 & 40 & 96 & 18.52 & 138 & 98 & 95 \\
III & 18.65 & 87 & 18.77 & 42 & 96 & 18.69 & 164 & 112 & 96 \\
\hline \hline
\end{tabular}



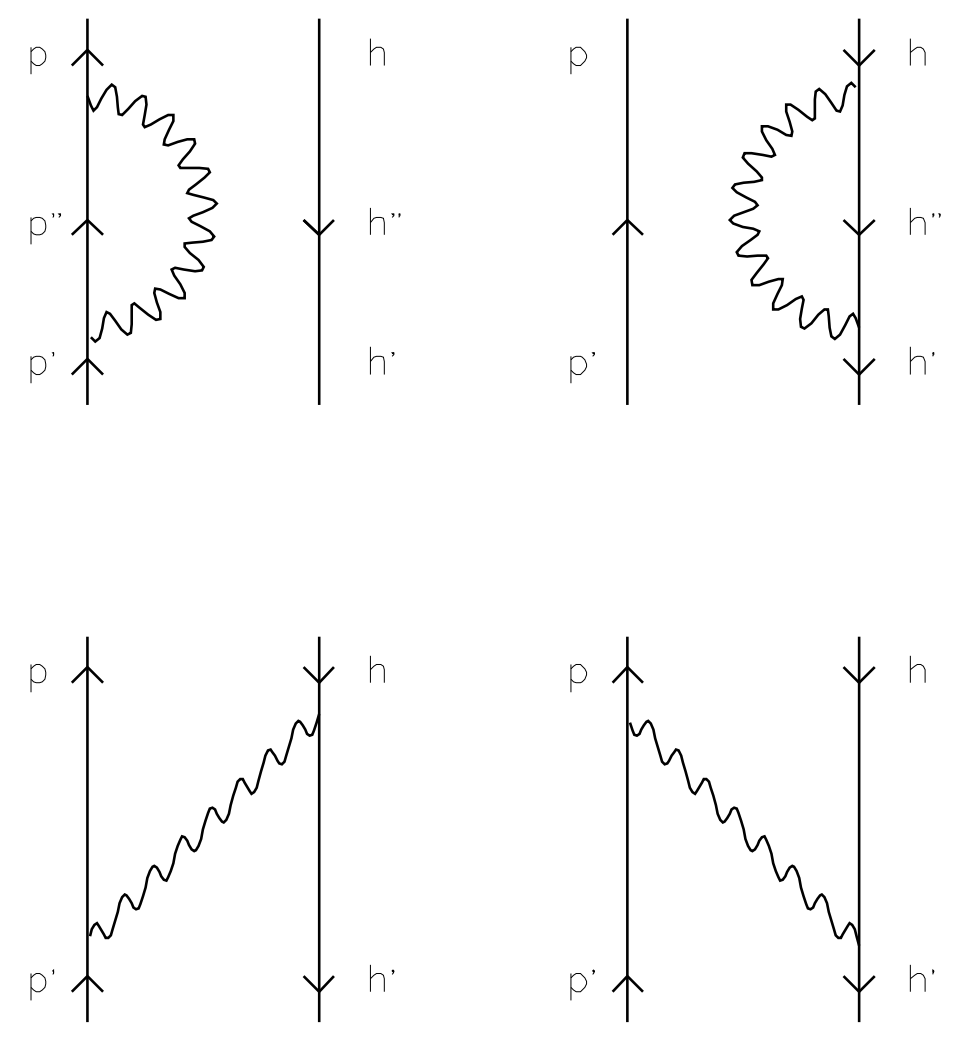